\title{
Improved Comprehensive Evaluation of Crohn's Disease Activity by Intestinal Computed Tomography Enterography Combined with Endoscopy and Biochemical Indicators
}

\section{yajun Liu}

Xiangya Hospital Central South University

feiyan tian

yiyang central hospital

jun yi

Xiangya Hospital Central South University

lingqi gong

Xiangya Hospital Central South University

lulu chen

Xiangya Hospital Central South University

shuijiao chen ( $D$ 4010383@csu.edu.cn )

Xiangya Hospital Central South University

xiaowei liu

Xiangya Hospital Central South University

Research article

Keywords: Crohn's disease, CT enterography, Activity index

Posted Date: September 15th, 2020

DOI: https://doi.org/10.21203/rs.3.rs-62368/v1

License: (c) (1) This work is licensed under a Creative Commons Attribution 4.0 International License. Read Full License 


\section{Abstract}

Background: The treatment goal for Crohn's disease (CD) has turned towards mucosal healing and resolution of the transmural inflammation. The purpose of this study was to evaluate how well computed tomography enterography (CTE) correlated with other treatments, in particular, endoscopy and biochemical indicators, in newly diagnosed patients with CD.

Methods: 123 patients with CD who received CTE in the Xiangya Hospital of Central South University, China, from April 2017 to April 2019 were included. Clinical case data and imaging results were retrospectively reviewed. The clinical activity of $C D$ was determined by the Crohn's disease activity index (CDAI) score and biochemical indicators. The relationships between CTE and endoscopy, CDAI score, and inflammatory markers were evaluated.

Results: The diagnostic rate of CTE in the 123 CD patients was 68.3\% (84/123). Logistic regression analysis showed that the specific CTE manifestations related to CD activity were intestinal wall stratification, comb sign, and abdominal abscesses $(P<0.05)$. Spearman's rank correlation analysis showed that inflammatory markers correlated positively with different CTE imaging manifestations. The CTE findings were consistent with colonoscopy results in 136 intestinal segments (73.1\%) in terms of lesion location but were not consistent in 50 intestinal segments (26.9\%).

Conclusion: intestinal CTE has a high degree of completion and is well tolerated, in contrast to endoscopy which is both invasive and unable to reflect the level of intestinal mucosal infiltration. In conclusion, intestinal CTE combined with endoscopy and biochemical indicators is both comprehensive and effective in evaluating the condition of $C D$ patients.

\section{Background}

Crohn's disease (CD) is a chronic granulomatous inflammatory bowel disease with unknown etiology. Although $\mathrm{CD}$ can involve the whole digestive tract, it more commonly involves only the terminal ileum. Intestinal inflammation develops gradually over time, eventually leading to severe intestinal dysfunction or even loss of intestinal function [1]. In recent years, the incidence of inflammatory bowel disease in Asia, especially in East Asia, has increased significantly, possibly because of the gradual westernization of lifestyle and industrialization [2-4]. The assessment of disease activity with stratification for CD patients is crucial for the determination of a therapeutic strategy and prognosis. Therefore, we urgently need an efficient method for comprehensively assessing CD.

Various indices are used to evaluate $C D$ activity including, for example, the Crohn's disease activity index (CDAI). However, CDAl has been questioned because of its subjectivity and complexity. It has been reported that in a randomized controlled study, the response rate of the CDAI score to placebo was as high as $33 \%[5,6]$. Studies have also shown that inflammation biomarkers such as C-reactive protein (CRP), the erythrocyte sedimentation rate (ESR), and fecal calprotectin are significantly correlated with endoscopic activity and the risk of $\mathrm{CD}$ recurrence. For example, if the CRP level continues to increase 
during treatment, the prognosis is likely to be poor $[7,8]$. It is well known that the risk of arteriovenous thrombosis in inflammatory bowel disease (IBD) patients is three times higher than that in normal subjects, which may be related to the increase of platelets and coagulation-related active substances (such as D-dimer and fibrinogen) in patients with active CD $[9,10]$. At present, endoscopic remission (ER) has often been used as the gold standard for evaluating the degree of inflammation in the intestinal mucosa. However, some studies have not observed a significant correlation between endoscopic mucosal lesions and clinical activity in $C D$. It is believed that $C D$, as a disease involving the whole intestinal wall, should be assessed by typical transmural inflammatory changes rather than superficial mucosal lesions [11]. In addition, since CD often affects the small bowel beyond the terminal ileum, ileo-colonoscopy alone may be inadequate for the correct evaluation of mucosal inflammation in CD.

In recent years, computed tomography enterography (CTE) has become an accepted non-invasive imaging technique to evaluate $\mathrm{CD}$ activity or postoperative recurrence. CTE can accurately reflect the distribution of lesions and inflammatory changes in the entire intestinal wall in an easy and semiquantitative manner. At the same time, CTE has the advantages of simplicity and good patient tolerance $[12,13]$. In addition, it is better able to dilate and observe the small intestine, especially the proximal small intestine, allowing an improved detection and diagnosis of high-order CD lesions, compared to enteroscopy. By 2008, CTE had surpassed endoscopy as the preferred examination method for CD [14]. The CTE signs have been well described and are closely associated with CD activity; these include mural stratification, mural hyperenhancement, bowel wall thickening, haziness of the surrounding mesenteric fat, and the comb sign.

Despite their widespread availability in clinical practice, the relative performances and relationships between CTE, biochemical indicators, and endoscopy as diagnostic tools to evaluate the therapeutic response in $C D$ have yet to be determined. This study was designed to determine the correlation between endoscopic disease activity, biochemical markers, and the CTE findings of inflammatory activity in diagnosed $C D$ patients, allowing a more accurate evaluation of $C D$ activity and providing new directions for the treatment of $C D$.

\section{Methods}

\subsection{Patient data}

A total of 123 consecutive patients who met the 2010 World Organization of Gastroenterology Practical Guidelines for the Diagnosis of Inflammatory Bowel Disease were enrolled in the study. Patients were 1865 years of age, not currently pregnant or nursing, and without any evidence of infectious colitis. Patients with a prior history of a bleeding disorder or blood-borne infections were excluded. The patients' past medical and surgical history was taken at the time of screening and a complete physical examination was performed at the same time. Blood and fecal samples for biomarker measurements were obtained at the time of screening. The CDAI score was determined by the sum of eight variables after adjustment with each weighting factor. Based on previous studies, the state of $C D$ activity was divided into two 
groups: disease remission (CDAl scores less than 150) and active disease (CDAl scores $₫ 150$ ). Measurements of the white blood cell count, platelet count, hemoglobin, hematocrit, CRP, and serum levels of total protein and albumin were also performed. The disease phenotype was determined according to the Montreal classification. Endoscopic lesions were assessed using the SES-CD.

\subsection{CTE technique and CTE image interpretation}

All CTE examinations were performed in our hospital, using a 64 row-multidetector CT Siemens Somaton Sensation scanner (Munich, Germany). Axial, coronal, and sagittal images $2 \mathrm{~mm}$ in thickness were obtained. Just before scanning, patients were asked to drink $4000 \mathrm{ml}$ of water containing $40 \mathrm{~g}$ of mannitol over $60 \mathrm{~min}$, at a steady rate of approximately $1000 \mathrm{ml}$ every $15 \mathrm{~min}$. Radiological interpretation and scoring were performed by two senior radiologists with more than eight years of experience dedicated to IBD imaging. They were blinded to all the clinical, laboratory, and endoscopic data and were asked to identify all bowel segments with signs of inflammation. When the diagnostic opinions were inconsistent, consensus was reached through consultation. Six CT signs of active CD (mural thickness, mural hyperenhancement, mesenteric fat proliferation, mesenteric fat densification, comb sign, and presence of strictures) were evaluated in ileocolonic segments. In addition, extraintestinal manifestations such as fistulas, abscesses, ascites, and lymphadenopathy were also recorded.

\subsection{Statistical Methods}

Categorical variables were expressed as percentages and compared by Fisher's exact test, and continuous variables were shown as means and standard errors of the mean (SEM). Logistic regression analysis and ROC curve analysis were used to screen the intestinal CTE manifestations of Crohn's disease in the active stage. Spearman correlation analysis was applied for assessing the correlations between CTE manifestations and biochemical indicators of inflammatory activity with $P<0.05$ taken as statistically significant. All data were arranged, processed, and analyzed with SPSS 17.0 software.

\subsection{Ethical review}

The study was reviewed and approved by the Ethics Committee of Xiangya Hospital of Central South University and was conducted according to the principles of the Declaration of Helsinki. All patients signed informed consent forms.

\section{Results}

\subsection{Population}

The CD patient demographics and clinical characteristics are shown in Table 1. A total of 123 newly diagnosed CD patients were enrolled. Ninety-four patients (76.4\%) were male while $29(23.6 \%)$ were female; there were thus significantly more male than female patients. The median age for diagnosis was 30 years-old (31.48 \pm 11.416 years). A total of 41 (33.3\%) patients had exclusive ileal involvement (L1) and a total of $62(50.4 \%)$ patients had ileocolonic involvement (L3), suggesting that the end of ileum was the most common site of involvement. It was found that the non-stenosis, non-penetration type (B1), and 
the stenosis type (B2) were the most common, with the penetration type (B3) being the least common. Specifically, the number of B2 type patients was 46 (37.4\%) but the number of B3 type patients was only $5(4.1 \%)$. The most common clinical manifestations of CD in patients were abdominal pain in 109 cases (88.6\%), diarrhea in 78 cases (63.4\%), and weight loss in 79 cases (64.2\%). Fever was more common in patients with active-stage disease (23 cases), and perianal lesions were the first manifestations in some patients. According to Best's CDAl score, 56 patients (45.5\%) were in the active stage (CDAl > 150) and 67 patients $(54.5 \%)$ were in the remission stage $(\mathrm{CDAl}<150)$. 
Table 1

Descriptive statistics of general clinical and laboratory data of CD patients

\begin{tabular}{|c|c|c|c|}
\hline & $\begin{array}{l}\text { Active stage } \\
\text { (CDAl囚150) }\end{array}$ & & $\begin{array}{l}\text { Remission stage } \\
(\mathrm{CDAl} \leq 150)\end{array}$ \\
\hline case(n) & 123 & 56 & 67 \\
\hline gender & $94(76.4)$ & $37(66.1)$ & $57(85.1)$ \\
\hline $\begin{array}{l}\text { male } \\
\text { famale }\end{array}$ & 29(23.6) & 19(33.9) & $10(14.9)$ \\
\hline age & $30(14-60)$ & & \\
\hline Course of disease(mouth) & 24(8days-240mouth) & & \\
\hline $\begin{array}{l}\text { Age at diagnosis } \\
\leq 40 \text { years } \\
₫ 40 \text { years }\end{array}$ & $\begin{array}{l}94(76.4) \\
29(23.6)\end{array}$ & $\begin{array}{l}41(73.2) \\
15(26.8)\end{array}$ & $\begin{array}{l}53(79.1) \\
14(20.9)\end{array}$ \\
\hline Location & $41(33.3)$ & $16(28.6)$ & 25(37.3) \\
\hline L1 & $9(7.3)$ & $7(12.5)$ & $2(3.0)$ \\
\hline L2 & $62(50.4)$ & $32(57.1)$ & $30(44.8)$ \\
\hline $\begin{array}{l}\text { L3 } \\
\text { L4 }\end{array}$ & $11(9.0)$ & $1(1.8)$ & $10(14.9)$ \\
\hline Behaviour & $46(37.4)$ & 18 & 28 \\
\hline B1 & $62(50.4)$ & 28 & 34 \\
\hline B2 & $5(4.1)$ & 2 & 3 \\
\hline B3 & $10(8.2)$ & 8 & 2 \\
\hline $\mathrm{B} 2+\mathrm{B} 3$ & & & \\
\hline
\end{tabular}




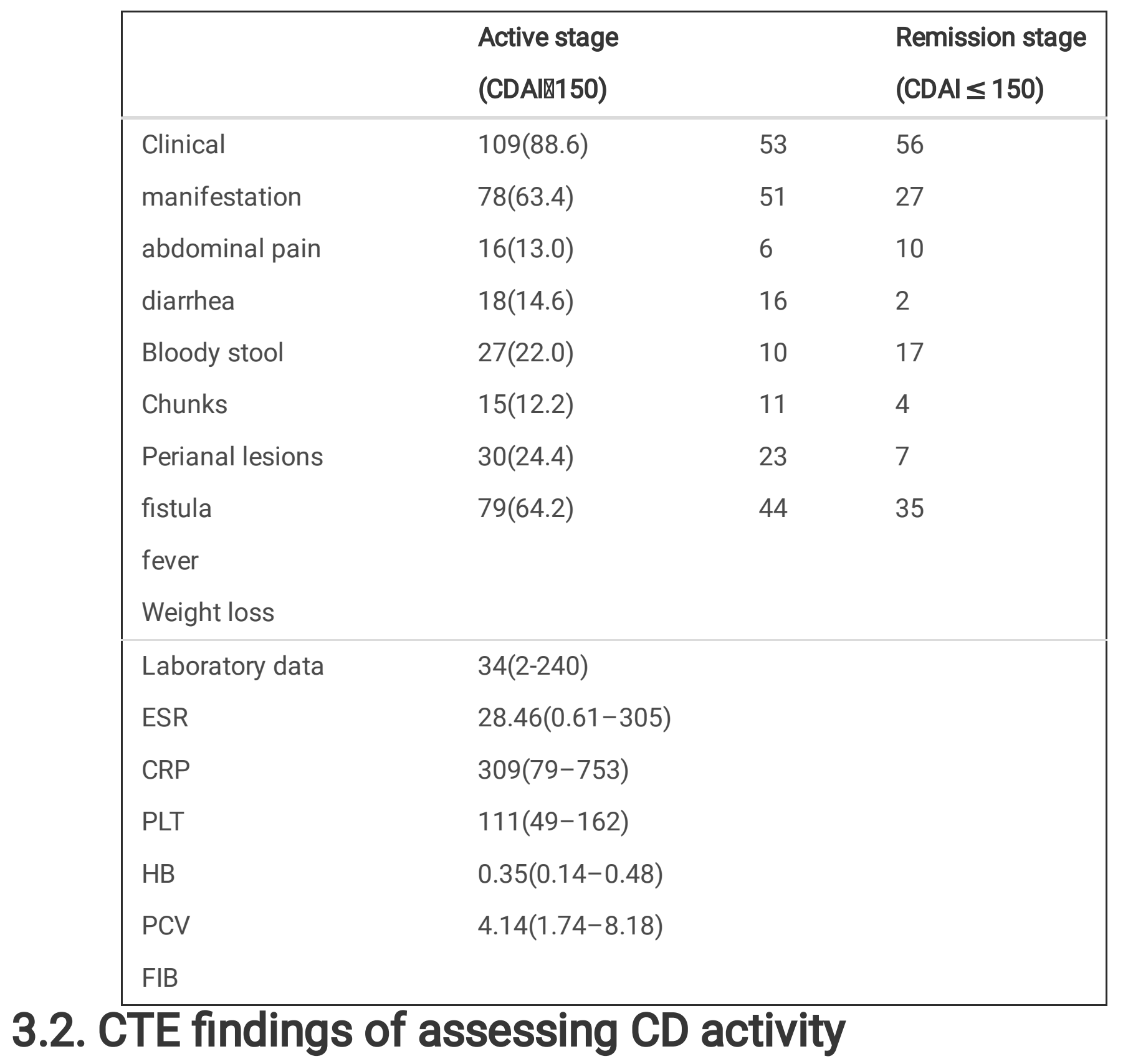

The CTE findings at baseline showed mural thickening in 107 patients (86.9\%) and hyperenhancement in 102 patients (82.9\%). A total of 58 patients (47.2\%) presented with the comb sign and 51 patients $(41.5 \%)$ presented with mural stratification. Strictures were observed in 60 patients (48.8\%), while fistulas were identified in seven patients $5.7 \%$ ) and abscesses in 11 patients (8.9\%). Univariate analysis of the intestinal CTE manifestations in active and remission CD patients is shown in Table 2. The Chi-square test showed that there were significant differences in intestinal wall thickening, intestinal stricture, intestinal wall stratification, comb sign, lipofibrosis, and the presence of abdominal abscesses between the active and remission stages of $C D(P<0.05)$. Among them, intestinal wall stratification, comb sign, and lipofibrosis showed significant differences $(P<0.01)$ (see Table 3 ) indicating the value of these parameters in judging the activity of $\mathrm{CD}$. 
Table 2

Univariate analysis of intestinal CTE manifestations in CD patients

\begin{tabular}{|c|c|c|c|}
\hline variables & $\begin{array}{l}\text { Remission stage } \\
\text { CDAI囚150 }\end{array}$ & $\begin{array}{l}\text { Active stage } \\
\text { CDAl } \geq 150\end{array}$ & $\begin{array}{l}\text { Univariate analysis } \\
P \text { value }\end{array}$ \\
\hline $\begin{array}{l}\text { Intestinal wall manifestations } \\
\text { bowel wall thickening }\end{array}$ & 54 & 53 & 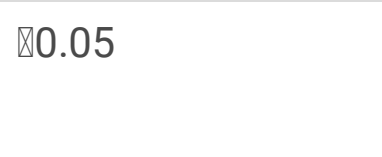 \\
\hline mucosal hyperenhancement & 53 & 49 & $\varangle 0.05$ \\
\hline stenosis & 26 & 34 & $\varangle 0.05$ \\
\hline Mural stratification & 9 & 42 & $\varangle 0.01$ \\
\hline $\begin{array}{l}\text { Periintestinalmanifestations } \\
\text { comb sign }\end{array}$ & 21 & 37 & $\varangle 0.01$ \\
\hline fibro-fatty proliferation & 31 & 42 & $\triangle 0.01$ \\
\hline Mesenteric lymphadenopathy & 21 & 17 & $\nabla 0.05$ \\
\hline ascites & 0 & 3 & $\nabla 0.05$ \\
\hline abdominal abscess & 2 & 9 & $\nabla 0.05$ \\
\hline fistula & 2 & 5 & $\varangle 0.05$ \\
\hline
\end{tabular}


Table 3

Logistic regression analysisof intestinal CTE manifestations in CD patients

\begin{tabular}{|llll|}
\hline variables & B & P & Odds ratio(95\%Cl) \\
\hline $\begin{array}{l}\text { Intestinal wall manifestations } \\
\text { bowel wall thickening }\end{array}$ & & 0.878 & - \\
\hline Mural hyperenhancement & & & \\
\hline stenosis & & 0.053 & - \\
\hline Mural stratification & 0.753 & - \\
\hline Periintestinalmanifestations & 1.417 & 0.005 & $4.126(1.519-11.206)$ \\
comb sign & & 0.000 & $21.294(7.617-59.526)$ \\
\hline fibro-fatty proliferation & & 0.779 & - \\
\hline Mesenteric lymphadenopathy & & 0.110 & - \\
\hline ascites & & 0.128 & - \\
\hline abdominal abscess & 2.321 & 0.019 & $10.186(1.471-70.513)$ \\
\hline fistula & & 0.657 & - \\
\hline
\end{tabular}

Furthermore, logistic regression analysis showed that there were significant differences in intestinal wall stratification, comb sign, and abdominal abscess between the active and remission stages $(P<0.05)$ (Table 3). However, the ROC curve analysis showed that while intestinal wall stratification has a relatively high diagnostic value for the assessment of CD activity, comb sign and lipofibrosis have relatively low diagnostic values.

\subsection{Analysis of correlation between CTE findings and biochemical indicators in CD patients}

As shown in Table 4, Spearman correlation analysis indicated that CRP was positively correlated with mural hyperenhancement, mural stratification, abdominal abscess, and comb sign. Nevertheless, ESR was closely related to intestinal wall thickening, mural hyperenhancement, and mural stratification, amongst others. Platelet counts (PLT) showed some correlation with intestinal wall thickening as well as mural hyperenhancement. However, hemoglobin was negatively correlated with intestinal wall thickening, intestinal wall stratification, and comb sign. It is, therefore, apparent that the CTE signs were significantly correlated with the biochemical indicators of inflammation, which has value in evaluating CD activity. 
Table 4

The correlation between CTE signs and inflammatory markers in CD

\begin{tabular}{|c|c|c|c|c|c|}
\hline & CRP & ESR & PLT & HB & FIB \\
\hline $\begin{array}{l}\text { Intestinal wall manifestations } \\
\text { bowel wall thickening }\end{array}$ & 0.172 & $0.029 \star \star$ & $0.183^{*}$ & $-0.237^{\star \star}$ & 0.138 \\
\hline mural hyperenhancement & $0.262^{\star \star}$ & $0.272^{\star \star}$ & $0.221^{*}$ & -0.041 & $0.197 *$ \\
\hline stenosis & 0.08 & 0.021 & 0.032 & -0.169 & 0.017 \\
\hline mural stratification & $0.364^{\star \star}$ & $0.318^{\star \star}$ & 0.117 & $-0.208^{*}$ & 0.162 \\
\hline $\begin{array}{l}\text { Periintestinal manifestations } \\
\text { comb sign }\end{array}$ & $0.181^{*}$ & 0.169 & 0.131 & $-0.297^{\star \star}$ & $0.313^{\star *}$ \\
\hline fibro-fatty proliferation & 0.175 & $0.28 * \star$ & 0.148 & -0.173 & 0.155 \\
\hline Mesenteric lymphadenopathy & 0.042 & 0.062 & -0.015 & -0.013 & 0.153 \\
\hline ascites & 0.023 & -0.131 & 0.018 & -0.132 & -0.107 \\
\hline abdominal abscess & $0.345^{\star \star}$ & $0.293^{\star \star}$ & 0.069 & -0.153 & $0.239 * *$ \\
\hline fistula & 0.127 & 0.144 & -0.073 & 0.124 & 0.117 \\
\hline
\end{tabular}

\subsection{Consistency of CTE findings and colonoscopy and in localization of lesions}

As can be seen from Table 5, during examinations to determine lesion localization, in 96 cases lesions were detected in the rectal colon by endoscopy. Among these, in 35 cases, lesions were found by CTE and endoscopy simultaneously and in 36 cases were not found by either CTE or endoscopy, suggesting that the two examination procedures have a high coincidence rate in the localization of colorectal lesions. There were 25 cases (26\%) with inconsistent agreement between the two examinations, including 20 cases with positive endoscopy but with negative CTE (Fig. 1a, b) and five cases with negative endoscopy but with positive intestinal CTE (Fig. 1c, d, e, f). Among them, intestinal wall thickening was found in five cases, irregular stenosis in two cases, and dilatation of the intestine in one case. 
Table 5

statistics of lesion location under enteroscopy and CT enterography

\begin{tabular}{|lllllllll|}
\hline colorectal & & \multicolumn{3}{c}{$\begin{array}{l}\text { terminal ileum } \\
\text { ileocecal valve }\end{array}$} & \multicolumn{3}{l|}{$\begin{array}{l}\text { Middle and upper ileum } \\
\text { Uppergastrointestinal tract }\end{array}$} \\
\hline endoscope & CTE & Cases & endoscope & CTE & Cases & endoscope & CTE & Cases \\
\hline+ & + & 35 & + & + & 46 & + & + & 9 \\
\hline- & - & 36 & - & - & 9 & - & - & 1 \\
\hline+ & - & 20 & + & - & 8 & + & - & 6 \\
\hline- & + & 5 & - & + & 10 & - & + & 1 \\
\hline
\end{tabular}

In addition, 73 cases had lesions that were localized to the terminal ileum and ileocecal valve by endoscopy. Of these, lesions were found by both CTE and endoscopy in 46 cases but neither CTE nor endoscopy detected lesions in nine cases, suggesting that both examination procedures have a high coincidence rate in the localization of colorectal lesions (75.3\%). There were 18 cases $(24.7 \%)$ where the results were inconsistent between the two methods. Of these, eight cases were positive on endoscopy but were negative on CTE (Fig. 2a, b). Ten cases with negative endoscopy were positive for CTE (Fig. 2c, d, e, f).

Furthermore, 17 cases of upper gastrointestinal tract lesions were detected by capsule endoscopy, transoral, or transanal enteroscopy in patients with $\mathrm{CD}$. Of these, nine cases were found with lesions of the upper gastrointestinal tract by CTE and endoscopy, and lesions were not identified by either method in one case. The localization of upper gastrointestinal lesions had a high coincidence rate (58.8\%) while seven cases (41.2\%) showed no coincidence. The results of endoscopy were positive but those of CTE were negative in six cases (Fig. 3aロb). Poor intestinal filling was identified in two cases, ulcers in six cases, strictures in two cases, and nodular protuberances in one case which were all negative on endoscopy. On the other hand, one case was only positive on CTE (Fig. 3c, d, e f) showing intestinal wall thickening.

\section{Discussion}

Recently, there has been a trend to avoid relying on clinical indices for determining CD treatment due to the lack of their correlation with endoscopic and histologic measures of disease activity $[15,16]$. According to the latest guidelines, the goal of CD treatment is histological evidence of mucosal healing, which is an objective indicator of the efficacy of $C D$ drugs and is related to the clinical recurrence rate and the reduction of surgical rate [17]. However, there are no accepted endoscopic criteria for mucosal healing at present. It is now essential to define the best way to monitor disease activity. Attempts to correlate outcomes with radiological signs of $\mathrm{CD}$ have produced variable findings. 
In this retrospective study, it was found that the radiologic findings of CTE correlated closely with CD activity. This is consistent with previous studies $[18,19]$. However, the importance of two CTE signs, namely, bowel wall thickening, and mural hyperenhancement, is still controversial, because the normal collapsed intestinal wall itself is thicker than the dilated intestinal wall and has a higher mucosal enhancement value, leading to uncertainty in the results. Interestingly, a prior study quantifying bowel wall thickening and mural hyperenhancement by semi-automated computer software found that the two parameters were significantly correlated with CD activity [20], providing a reference for future research.

Our study found that, compared with mural hyperenhancement, mural stratification is likely to be a more valuable measure reflecting the activity of $C D$, especially when it is caused by edema [12]. It is worth noting that the specificity of mural stratification is very poor which can be seen in other types of intestinal inflammation such as ischemic bowel disease. However, as the patients in this study were clinically or pathologically confirmed CD patients, the poor specificity did not affect the results. In addition, the comb sign is another commonly recognized CTE sign reflecting CD activity. In a quantitative study of the comb sign [21], it was found that the comb sign score increased significantly in the CD group and predicted CD activity with an accuracy of up to $80 \%$ when the appropriate cut-off point was found. Besides, some studies suggest that patients with a typical comb sign may require more hospitalization and invasive treatment, and are more likely to have longitudinal ulcers and extensive organ damage [22], which further demonstrates the importance of the comb sign in evaluating $C D$ condition and provides a reference for more active treatment. In addition, Colombel et al [23] considered that the increase of peri-intestinal fat density was a specific imaging sign of active-stage Crohn's disease, reflecting the inflammatory infiltration of peri-mesenteric fat mediated by mesenteric adipocytes. However, one of the CT signs included in the present study is peri-intestinal adipose fibrosis, although its specificity is very poor in relation to the present results. Nevertheless, the ROC curve analysis shows that it has a low diagnostic significance and, therefore, deserves proper attention.

To date, numerous biomarkers related to inflammatory activity have been described but these depended heavily on clinical or endoscopic assessment as references. There are few studies on the relationship between CT imaging findings and biochemical indicators. Our study found that CTE signs, such as mural stratification and the comb sign, are significantly correlated with inflammatory biochemical indicators. However, this view is controversial at present. Minordi et al showed that the comb sign and intestinal wall thickening were positively correlated with CRP [24] while in another quantitative study of the comb sign, it was considered that the comb sign is not related to CRP [21]. In addition, ESR is also considered to be a useful indicator for monitoring CD activity, but its role has not been fully defined. Some studies have suggested that the comb sign has a good correlation with ESR [24], but other studies have not confirmed these findings. Previous studies have suggested that both the comb sign and lymphadenopathy are correlated with the platelet count rather than fibrinogen. In addition, the platelet count has been reported to be related to CRP $[25,26]$. As mentioned above, one-third of IBD patients have iron deficiency anemia which has been reported to be related to the platelet count. Controlling inflammation and improving anemia may reverse the trend of platelet increase, suggesting that the degree of anemia may be related to CD activity. Fecal calcium protein (FC) has been widely studied in IBD [27-29] and has proved to be a 
valuable predictor of disease relapse and recurrence. Susana and his team recently found that FC performed better than CRP in predicting endoscopic activity and was significantly correlated with the CTE findings [30].

Although previous studies have shown that the severity of endoscopic mucosal lesions is not significantly correlated with the CDAI and blood biochemical parameters, the importance of endoscopic mucosal healing is increasingly recognized [18] as this results in a better prognosis. The CD activity index under capsule endoscopy has been shown in numerous studies to be correlated with intestinal wall thickening, mucosal enhancement, and parenteral manifestations [31]. Interestingly, the current study suggests that only intestinal wall thickening is associated with endoscopic results. All 123 patients successfully completed CT enterography, indicating that this examination can make up for the limitations of enteroscopy and allows improved completion and tolerance. However, the results of this study suggest that CTE is less sensitive in the diagnosis of intestinal stenosis. This may be related to the characteristics of the CT tomographic scan. The thinnest slice of the 64-slice spiral CT scan used in our hospital is $5 \mathrm{~mm}$, while the distribution of intestinal stenosis is limited, and the sensitivity of CTE to intestinal peristalsis is poor, which may result in a missed diagnosis of the narrow segment during the scanning process.

However, one complication of the use of CTE to monitor CD is that it may lead to radiation damage. Fortunately, there are several strategies available to reduce radiation exposure without affecting diagnostic accuracy. In this study, the sample size was relatively small and further studies with a larger sample size should be conducted to confirm the findings. Another limitation is that we have only utilized CTE to evaluate CD activity. Magnetic resonance enterography (MRE) has emerged as a nonionizing alternative to CTE and we intend to compare the differences between CTE and MRE in assessing CD activity.

In conclusion, we have found that, while CTE, endoscopy, and biochemical markers have limitations, all are nevertheless good markers for the evaluation of CD activity. Only when these tools are used comprehensively and in combination, can we judge CD activity more accurately to guide future treatment.

\section{Abbreviations}

Crohn's disease:CD ; Computed tomography enterography:CTE ; Crohn's disease activity index:CDAl ; Creactive protein:CRP; Erythrocyte sedimentation rate:ESR ; Inflammatory bowel disease: IBD

\section{Declarations}

Acknowledgements: Not applicable.

Funding: This study was funded by the National Natural Science Foundation of China(81700464). The funders had a role in study design, decision to publish and preparation of the manuscript. No additional external funding was received for this study. 
Availability of data and materials

The datasets used and/or analyzed during the current study are available from the corresponding author upon reasonable request.

\section{Authors' contributions}

YJ L and FY T draft the manuscript. J Y and LQ G collected clinical data. LL C revised the manuscript. SJ $\mathrm{C}$ and XW L provided general advice on the case and supervised the study. All authors read and approved the final manuscript.

\section{Ethics approval and consent to participate}

This study was approved by the Medical Ethical Committee of the XiangYa Hospital Central South University. All patients had signed informed consent.

\section{Statement of Ethics}

Subjects (or their parents or guardians) have given their written informed consent to publish their case (including publication of images).

\section{Consent for publication}

Written informed consent was obtained from the patient for the publication of this aticle.

\section{Competing Interests}

The authors declare that they have no competing interests.

\section{References}

1. Cosnes J, Bourrier A, Seksik P. Factors affecting outcomes in Crohn's disease over 15 years. Gut. 2012;61(8):1140-5.

2. Zhulina Y, Udumyan R, Halfvarson J. The changing face of Crohn's disease: a population-based study of the natural history of Crohn's disease in Örebro, Sweden 1963-2005. Scand J Gastroenterol. 2016;51(3):304-13.

3. Hommes D, Colombel JF, Sandborn WJ. Changing Crohn's disease management: need for new goals and indices to prevent disability and improve quality of life. J Crohns Colitis. 2012;6(Suppl 2):224-34.

4. Duricova D, Fumery M, Gower-Rousseau C. The natural history of Crohn's disease in children: a review of population-based studies. Eur J Gastroenterol Hepatol. 2017;29(2):125-34.

5. Tinè F, Rossi F, Sferrazza A, et al. Meta-analysis: remission and response from control arms of randomized trials of biological therapies for active luminal Crohn's disease. Aliment Pharmacol Ther. 2008;27(12):1210-23. 
6. Novak G, Parker CE, Pai RK, et al. Histologic scoring indices for evaluation of disease activity in Crohn's disease. Cochrane Database Syst Rev. 2017;7:CD012351.

7. Tang J, Gao X, Zhi M, et al. Plateletcrit: a sensitive biomarker for evaluating disease activity in Crohn's disease with low hs-CRP. J Dig Dis. 2015;16(3):118-24.

8. Björkesten CG, Nieminen U, Färkkilä M. Mucosal healing at 3 months predicts long-term endoscopic remission in anti-TNF-treated luminal Crohn's disease. Scand J Gastroenterol. 2013;48(5):543-51.

9. Iannone A, Ruospo M, Palmer SC, et al. Systematic review with network meta-analysis: endoscopic techniques for dysplasia surveillance in inflammatory bowel disease. Aliment Pharmacol Ther. 2019.

10. Voudoukis E, Karmiris K, Oustamanolakis P, et al. Association between thrombocytosis and iron deficiency anemia in inflammatory bowel disease. Eur J Gastroenterol Hepatol. 2013;25(10):1212-6.

11. Annese V, Dapemo M, Rutter MD. European evidence based consensus for endoscopy in inflammatory bowel disease. J Crohns Colitis. 2013;7(12):982-1018.

12. Kim SH. Computed tomography enterography and magnetic resonance enterography in the diagnosis of Crohn's disease. Intest Res. 2015;13(1):27-38.

13. Lo Re G, Cappello M, Tudisca C, et al. CT enterography as a powerful tool for the evaluation of inflammatory activity in Crohn's disease: relationship of CT findings with CDAl and acute-phase reactants. Radiol Med. 2014;119(9):658-66.

14. Sipponen T, Nuutinen H, Färkkilä M. Endoscopic evaluation of Crohn's disease activity: comparison of the CDEIS and the SES-CD. Inflamm Bowel Dis. 2010;16(12):2131-6.

15. Alkim H, Ayaz S, Sahin B. Continuous active state of coagulation system in patients with nonthrombotic inflammatory bowel disease. Clin Appl Thromb Hemost. 2011;17(6):600-4.

16. Miranda García P, Chaparro M, Gisbert JP. [Evaluation of the concordance between biological markers and clinical activity in inflammatory bowel disease]. Med Clin (Barc). 2015;144(1):9-13.

17. Baert F, Moortgat L, Van Assche G, et al. Mucosal healing predicts sustained clinical remission in patients with early-stage Crohn's disease. Gastroenterology. 2010;138(2):463-8. quiz e10-1.

18. Cellier C, Sahmoud T, Froguel E, et al. Correlations between clinical activity, endoscopic severity, and biological parameters in colonic or ileocolonic Crohn's disease. A prospective multicentre study of 121 cases. The Groupe d'Etudes Thérapeutiques des Affections Inflammatoires Digestives. Gut. 1994;35(2):231-5.

19. Daperno M, D'Haens G, Van Assche G, et al. Development and validation of a new, simplified endoscopic activity score for Crohn's disease: the SES-CD. Gastrointest Endosc. 2004;60(4):505-12.

20. Bodily KD, Fletcher JG, Solem CA, et al. Crohn Disease: mural attenuation and thickness at contrastenhanced CT Enterography-correlation with endoscopic and histologic findings of inflammation. Radiology. 2006;238(2):505-16.

21. Wu YW, Tao XF, Miao F. Quantitative measures of comb sign in Crohn's disease: correlation with disease activity and laboratory indications. Abdom Imaging. 2012;37(3):350-8. 
22. Lee SS, Ha HK, Yang SK, et al. CT of prominent pericolic or perienteric vasculature in patients with Crohn's disease: correlation with clinical disease activity and findings on barium studies. AJR Am J Roentgenol. 2002;179(4):1029-36.

23. Colombel JF, Solem CA, Sandborn WJ, et al. Quantitative measurement and visual assessment of ileal Crohn's disease activity by computed tomography enterography: correlation with endoscopic severity and C reactive protein. Gut. 2006;55(11):1561-7.

24. Minordi LM, Vecchioli A, Bonomo L. CT findings and clinical activity in Crohn's disease. Clin Imaging. 2009;33(2):123-9.

25. Kapsoritakis AN, Koukourakis MI, Sfiridaki A, et al. Mean platelet volume: a useful marker of inflammatory bowel disease activity. Am J Gastroenterol. 2001;96(3):776-81.

26. Solem CA, Loftus EV Jr, Sandborn WJ. Correlation of C-reactive protein with clinical, endoscopic, histologic, and radiographic activity in inflammatory bowel disease. Inflamm Bowel Dis. 2005;11(8):707-12.

27. Lasson A, Simrén $M$, Strid $H$. Fecal calprotectin levels predict the clinical course in patients with new onset of ulcerative colitis. Inflamm Bowel Dis. 2013;19(3):576-81.

28. Ferreiro-Iglesias R, Barreiro-de Acosta M, Dominguez-Muñoz JE. Accuracy of Consecutive Fecal Calprotectin Measurements to Predict Relapse in Inflammatory Bowel Disease Patients Under Maintenance With Anti-TNF Therapy: A Prospective Longitudinal Cohort Study. J Clin Gastroenterol. 2018;52(3):229-34.

29. Ferreiro-Iglesias R, Barreiro-de Acosta M, Otero Santiago M, et al. Fecal Calprotectin as Predictor of Relapse in Patients With Inflammatory Bowel Disease Under Maintenance Infliximab Therapy. J Clin Gastroenterol. 2016;50(2):147-51.

30. Lopes S, Andrade P, Afonso J, et al. Monitoring Crohn's disease activity: endoscopy, fecal markers and computed tomography enterography. Therap Adv Gastroenterol. 2018;11:1756284818769075.

31. Tong JL, Feng Q, Shen J, et al. Computed tomography enterography versus balloon-assisted enteroscopy for evaluation of small bowel lesions in Crohn's disease. J Gastroenterol Hepatol. 2013;28(7):1180-6.

\section{Figures}



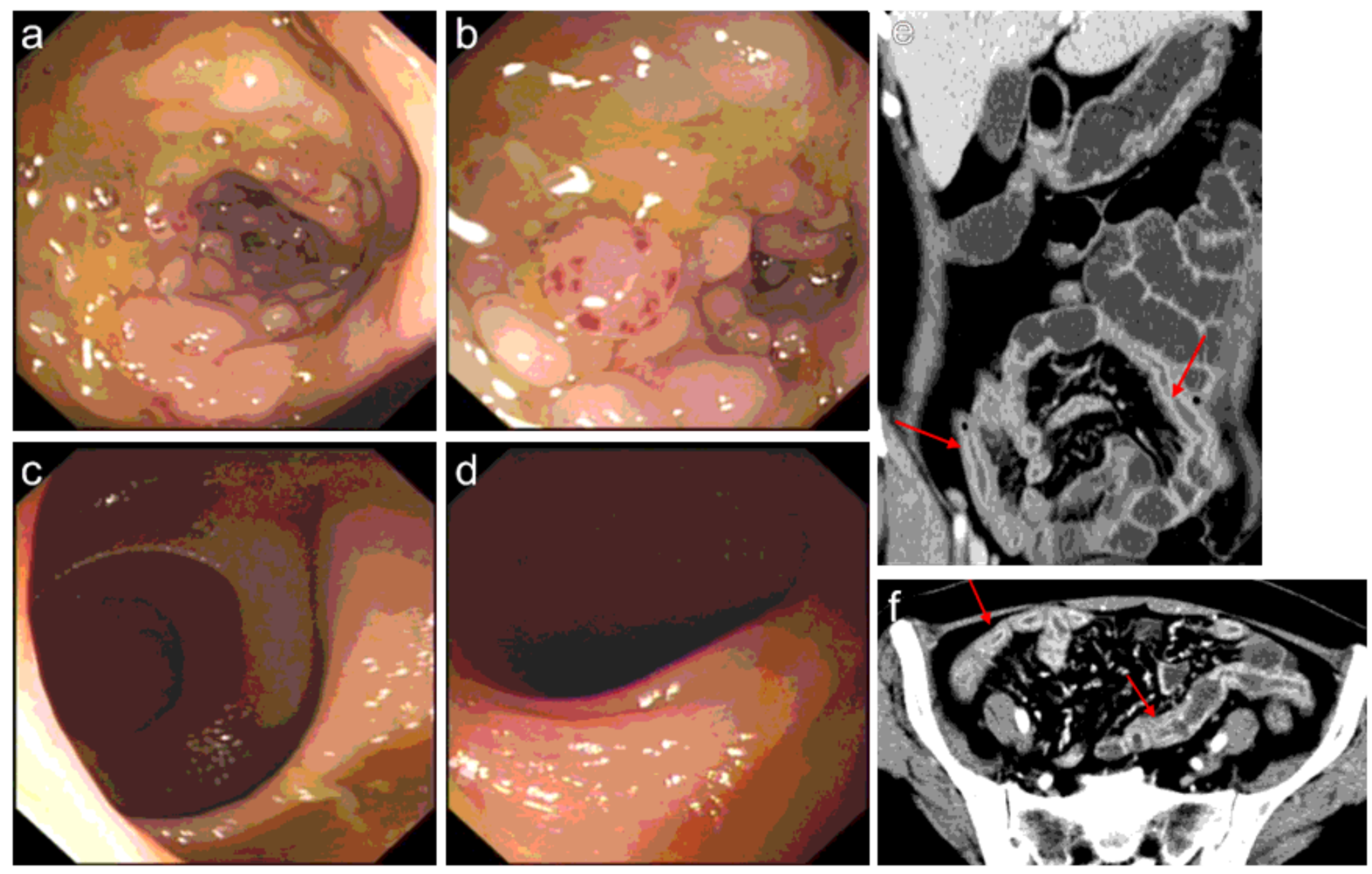

\section{Figure 1}

Endoscopic findings at the ascending colon $(a, b)$ and corresponding computed tomography enterography images (e). Endoscopic findings at the ascending colon $(c, d)$ and corresponding computed tomography enterography images $(f)$. 

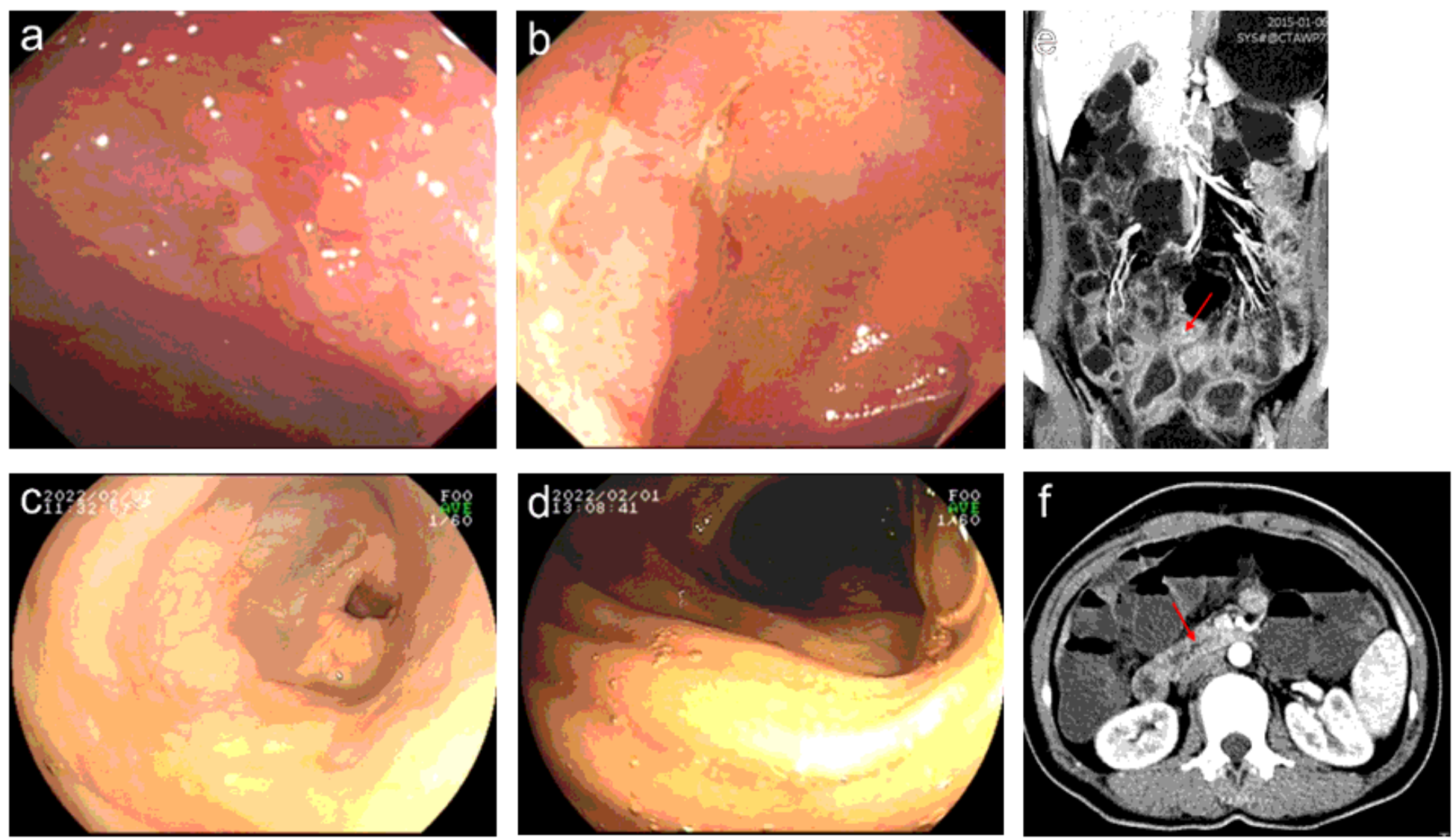

Figure 2

Endoscopic findings at the terminal ileum $(a, b)$ and corresponding computed tomography enterography images (e). Endoscopic findings at the ileocecal valve (c, d) and corresponding computed tomography enterography images $(\mathrm{f})$.
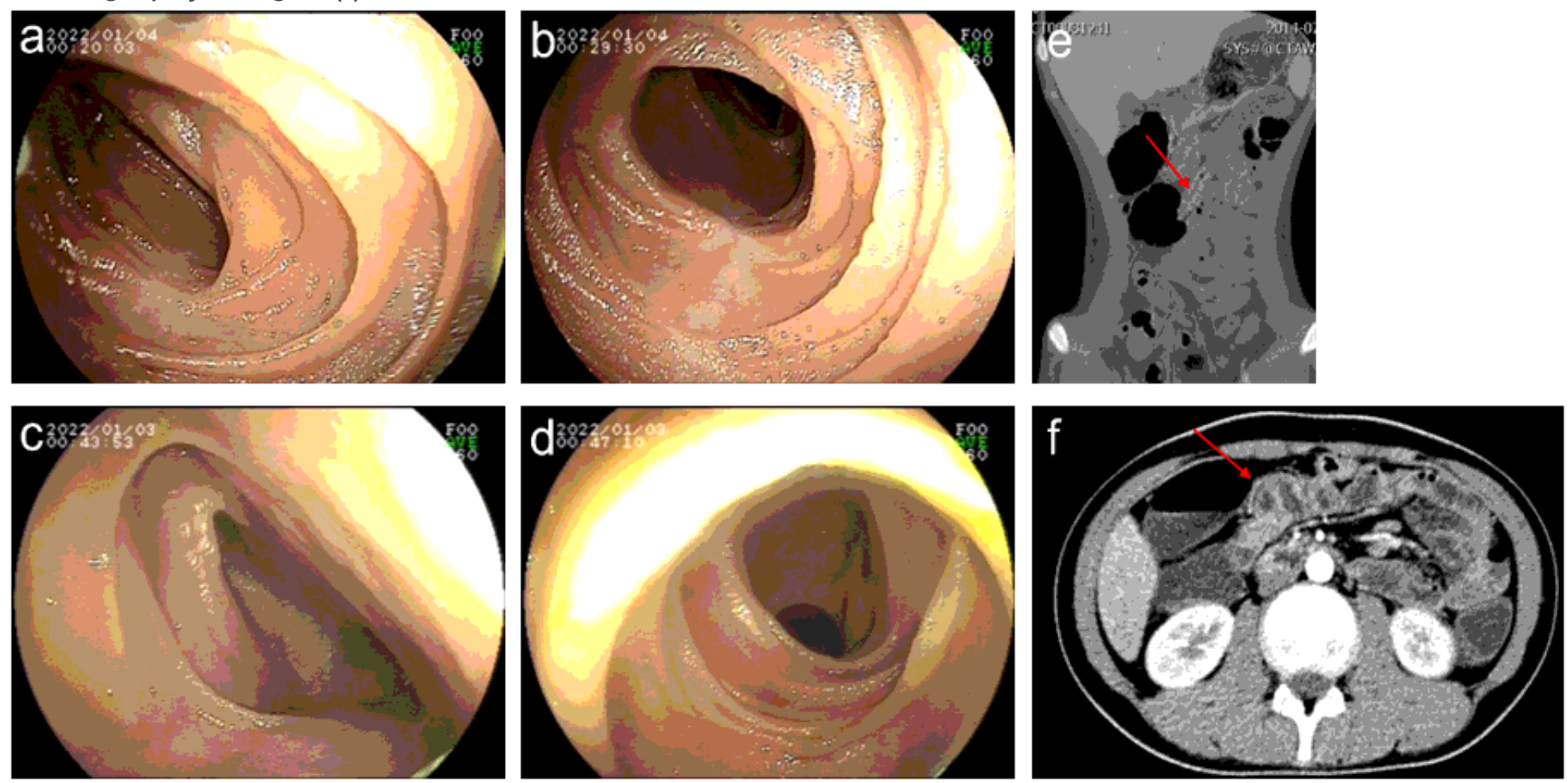


\section{Figure 3}

Endoscopic findings at the middle and lower jejunum $(a, b)$ and corresponding computed tomography enterography images (e). Endoscopic findings at the mid-upper ileum (c, d) and corresponding computed tomography enterography images (f). 